\title{
Phase separation in lithium intercalated anatase: A theory
}

\author{
O.V.Velychko, I.V.Stasyuk \\ Institute for Condensed Matter Physics of the National Academy of Sciences of Ukraine, \\ 1 Svientsitskii Str., 79011 Lviv, Ukraine
}

Received May 25, 2009, in final form May 26, 2009

\begin{abstract}
Lithium intercalated anatase used in Li-ion batteries has some special features: coexistence of Li-rich and Lipoor phases as well as two possible positions for $\mathrm{Li}$ ions in the oxygen tetrahedron. A theoretical description of the compound considering those peculiarities is presented. As shown by the performed symmetry analysis, the intercalation induced lattice deformation can be accompanied by the ordering of antiferroelectric type (internal piezoeffect). In the following step, a qualitative illustration of the phase separation in the lithiated anatase is given within the Landau expansion at the proper choice of coefficients. A microscopic model for description of the compound is also proposed which combines features of the Mitsui and Blume-Emery-Griffits models and utilizes the symmetry analysis results. Various ground state and temperature-dependent phase diagrams of the model are studied to find a set of model parameters corresponding to the lithiated anatase. A phase separation into the empty and half-filled phases in a wide temperature range has been found closely resembling the phase coexistence in the intercalated crystal. In the framework of the model, the two-position $\mathrm{Li}$ subsystem could have the ordering of ferro- or antiferroelectric types which, however, has not been yet observed by the experiment.
\end{abstract}

Key words: anatase, intercalation, lithium, phase separation, Landau expansion, lattice model

PACS: $71.20 . T x, 64.60 . \mathrm{Cn}, 64.60 . \mathrm{De}$

\section{Titania in a nutshell}

\subsection{Titanium dioxide: polymorphs, properties and applications}

There are eleven known polymorphs of titanium dioxide (titania). The most common natural forms (rutile, anatase and brookite) are just different space arrangements of the $\mathrm{TiO}_{6}$ group where a titanium cation is located in the centre of the slightly deformed octahedron shaped by six oxygen anions. Each polymorph has its own deviations from the ideal octahedron but it is always elongated along the certain axis. Thus, two (apical) oxygens are further away from the titanium than four others (equatorial).

Being the most stable polymorphs, rutile and anatase are widely used and intensively studied. They are very similar in many details (e.g. the arrangement of atoms and average lengths of bonds) [1]. However, anatase is $10 \%$ less dense than rutile and an additional volume is condensed in the voids affecting the cell averaged properties such as compressibility and dielectric constant. Moreover, this minor difference becomes crucial at crystal intercalation. In both polymorphs, an elementary cell consists of two formula units but unlike the tetragonal rutile $\left(P 4_{2} / \mathrm{mnm}\right)$ the standard crystallographic cell of body-centered tetragonal anatase $\left(I 4_{1} / a m d\right)$ is chosen to consist of two elementary cells.

The titanium atoms, and hence, the octahedra, are arranged in such a way that each oxygen is at the same time an equatorial atom for one titanium, and an apical one for the other titanium atom in the same unit cell. Neighboring octahedra are sharing edges and corners with each other. Two and four edges of each octahedron are shared in rutile and anatase, respectively. The basic octahedra are distorted in such a way that each shared edge is shortened, the other edges being correspondingly elongated. The shortened oxygen-oxygen bonds are often named as the bridge bonds (in the sense that it bridges the interaction between Ti ions: metal-oxygen-metal). In rutile 
the bridge bond connects two equatorial oxygen atoms. Hence, the octahedra form the vertical linear chains. The octahedra belonging to adjacent chains are connected only through one corner: an oxygen atom which is both apical and equatorial for the two touching octahedra. In anatase, the octahedra are arranged in order to share a diagonal edge between an apical and an equatorial atom. Thus, octahedra form zig-zag chains orthogonal to the crystallographic axis. There are two sets of chains orthogonal to each other, that are connected through a common octahedron.

The list of hi-tech applications of titanium dioxide is quite impressive. They are primarily related to its photoactivity. For example, $\mathrm{TiO}_{2}$, particularly in the anatase form, is a photocatalyst under ultraviolet light. The strong oxidative potential of the positive holes oxidizes water to create hydroxyl radicals (the Honda-Fujishima effect [2]).

Superhydrophilicity phenomenon for glass coated with titanium dioxide is caused by ultraviolet light partially removing oxygen atoms from the surface of the titanium oxide. The areas where oxygen atoms were removed became hydrophilic, while the same size areas where no oxygen atoms were taken away turned out to be hydrophobic [3]. The result is a $\mathrm{TiO}_{2}$-coated glass which is antifogging and self-cleaning.

Dielectric properties of titanium dioxide distinguish it as semiconductor [4] (to stress the difference: anatase is semiconductor of the n-type while rutile is of p-type which is utilized in the gas sensor [5]). Due to its high dielectric constant, it is commonly used as a dielectric in electronic devices, such as thin film capacitors [6] and MOS devices [7], as well as for the fabrication of anti-reflection coatings, interference filters [8], as well as optical wave-guides [9].

Although both rutile and anatase are potentially interesting for photo-catalysis and photoelectrochemical applications, experimental investigations have mostly focused on the more prospective anatase polymorph. It has a wider optical-absorption gap and a smaller electron effective mass which presumably leads to a higher mobility for the charge carriers [10] and plays a key role in the injection process of novel dye-sensitized photochemical solar cells with high conversion efficiency [11]. In some cases, the two materials are used together in the same device, exploiting their peculiar properties for different purposes. For example, a typical low-cost photo-voltaic module is composed of a transparent conducting photo-electrode of dye-sensitized nanocrystalline anatase, a spacer of electrically insulating, light-reflecting particles of rutile, and a counter-electrode of graphite powder [12]. Hence, anatase is used due to its efficient coupling with the dye, and rutile for its high dielectric constant.

Furthermore, the open crystallographic structure of anatase facilitates the accommodation of substantial amounts of small ions ( $\mathrm{Li}, \mathrm{H}$, etc.) within the lattice. Lithium insertion changes the optical properties of $\mathrm{TiO}_{2}$ : it turns the white powder dark blue whilst in thin film form it changes from being transparent to partially reflecting (electrochromism) which is used in displays and sunblinds (switchable mirrors). Combining an electrochromic film and a photovoltaic film to form the two electrodes of an electrochemical cell one can achieve a photochromic structure [13].

\subsection{Intercalation of rutile and anatase: experiment and theory}

In the last decades the Li-ion batteries have run into operation as a result of their high energy capacity, re-chargeability and environmentally friendly properties. Anatase $\mathrm{TiO}_{2}$ may act as an anode in such a battery [14]. In practice, anatase is not the ideal candidate because of its relatively low potential versus other electrode materials. Better properties are demonstrated, e.g. by similar compounds with the spinel structure $\mathrm{LiTi}_{2} \mathrm{O}_{4}[15], \mathrm{Li}_{4} \mathrm{Ti}_{5} \mathrm{O}_{12}$ [16] and their manganesian analogues $\mathrm{LiMn}_{2} \mathrm{O}_{4}$ and $\mathrm{LiMg}_{0.1} \mathrm{Ni}_{0.4} \mathrm{Mn}_{1.5} \mathrm{O}_{4}$ [17]. Here anatase is considered as a well defined model material displaying many typical properties of transition metal oxide electrodes. The electrode and electrochromic properties of lithiated anatase are already well documented and partly exploited commercially. However, the impressive experimental breakthrough in the study of the microscopic processes resulting in these achievements is not accompanied by theoretical investigations.

As mentioned earlier, anatase $\mathrm{TiO}_{2}$ has a body-centered tetragonal structure indexed by the $I 4_{1} /$ amd space group. Upon lithiation, anatase lattice undergoes an orthorhombic distortion that results in the $\mathrm{Li}_{0.5} \mathrm{TiO}_{2}$ phase (sometimes referred to as Li-titanate) indexed by the space group Imma, where the fourth order axis is lost due to the distortion in the $a b$ plane [18]. The change 
in symmetry is accompanied by a decrease of the unit cell along the $c$-axis and by an increase along the $b$-axis, resulting in a $4 \%$ increase of the unit cell volume. Lithium was found to reside in the interstitial voids within the oxygen octahedra [18]. The structural change can be explained as occupation of $\mathrm{Ti}-\mathrm{Ti}$ bonding atomic orbitals by the electron that enters the $\mathrm{TiO}_{2}$ lattice with each Li-ion to maintain charge neutrality.

In comparison with the number of experimental studies of intercalated titanium dioxide the list of theoretical works on the subject looks very short containing primarily $a b$ initio approaches. Some experimentally established properties are fairly well explained but some predictions are not confirmed by experiment.

Already in the pioneer work [19] a higher possibility of lithium intercalation in the anatase structure than in rutile was predicted as well as the absorption energies obtained were calculated and Li-induced local one-electron energy levels were found in the gap between the upper valence band and the conduction band and could be attributed to $\mathrm{Ti}^{3+}$ states.

The calculations of the relative lithium insertion potentials were performed for the rutile, anatase, brookite, ramsdellite, colombite, spinel, and orthorhombic polymorphs of titanium dioxide from the first principles periodic Hartree-Fock approach [20] also indicating that lithium was completely ionized in $\mathrm{LiTiO}_{2}$ and that the charge transfer is predominantly to the oxygen sublattice. A similar study of the average voltage to intercalate lithium in various metal oxides (among them $\mathrm{TiO}_{2}$ ) and dichalcogenides was performed utilizing the $a b$ initio pseudopotential method [21]. It was also found that Li was fully ionized in the intercalated compounds but with its charge distributed among the anion and the metal.

A series of works [22-28] should be mentioned, where the lithium intercalation in both rutile and anatase was $a b$ initio modelled taking into account thermodynamic and kinetic effects. The important role of strong local deformations of the lattice and elastic screening of interlithium interactions was established, the absence of insertion into rutile at room temperature was explained in terms of inaccessibility of the low-energy configurations due to highly anisotropic diffusion, a phase separation in anatase into a Li-rich phase and a Li-poor phase was described and the existence of a new distorted rock-salt phase for $\mathrm{LiTiO}_{2}$ was predicted $[22,28]$. The calculated open circuit voltage profile reproduced and explained the characteristic features of experimental discharge curves for both polymorphs [24]. An analysis of the site preference for Li intercalation in rutile and diffusion pathways of ions was performed. The expansion of the host structure on $\mathrm{Li}$ insertion was found to contribute to the enhanced diffusion of Li ions along the $c$ direction while a large distortion of the rutile framework nearly suppressed Li diffusion in the $a b$ planes; computed diffusion coefficients were found in excellent agreement with the measured values [26]. A new phase of $\mathrm{LiTiO}_{2}$ is predicted which may be accessed through electrochemical lithiation of ramsdellite-structured $\mathrm{TiO}_{2}$ at the lowest potential (remaining constant over a wide range of Li concentrations) reported for titanium dioxide based materials [29].

$A b$ initio study of the elastic properties of single and polycrystal $\mathrm{TiO}_{2}$ and other IV-B group oxides in the cotunnite structure was performed [30].

Up till now only one non- "ab initio" description of the intercalation in titanium dioxide by means of the pseudospin-electron model [31] (where the pseudospin formalism was used in describing the intercalant subsystem) was performed. The possibility of the phase transitions accompanied by an abrupt change of the concentration of intercalated ions and a significant increase of electrostatic capacity of the system was predicted.

\subsection{Special features of the lithium intercalated anatase: phase equilibrium and double positions for lithium ions}

Upon lithium insertion, an increasing fraction of the material changes its crystallographic structure from anatase $\mathrm{TiO}_{2}$ to Li-rich lithium titanate $\mathrm{Li}_{0.6} \mathrm{TiO}_{2}$ (sometimes a different stoichiometry is reported: $\mathrm{Li}_{0.5} \mathrm{TiO}_{2}$ or $\mathrm{Li}_{0.55} \mathrm{TiO}_{2}$; as will be shown below it depends on the size of $\mathrm{TiO}_{2}$ crystallites). Phase separation occurs on the Li-rich and the $\mathrm{Li}$-poor $\left(\mathrm{Li}_{0.01} \mathrm{TiO}_{2}\right)$ phases [32].

Such a two-phase equilibrium system in the electrodes provides a constant electrical potential between their electrodes (so-called plateau in potential on the discharge curve) for a wide range of 
the lithium concentration, because only the relative phase fractions vary on charging (or discharging) the lithium while their stoichiometries remain unchanged [33]. The Li-rich lithium titanate phase progressively moves inside the anatase electrode as a front parallel to the interface and returns during lithium extraction exactly in the way it came in [34].

Both in the anatase and in the lithium titanate lattice, $\mathrm{Li}$ is found to be hopping over the available sites with activation energies of 0.2 and $0.09 \mathrm{eV}$, respectively. However, macroscopic intercalation data show activation energies of $0.5 \mathrm{eV}$ because the diffusion through the phase boundary determines the activation energy of the overall diffusion and the overall diffusion rate itself [32].

Recent NMR spectroscopy study [35] of nanosized lithiated anatase revealed further important details of the phase behavior and morphology. The coexistence of the Li-poor and the Li-rich phases is possible only in the particles of the size exceeding $120 \mathrm{~nm}$ due to the surface strain (occurring between the phases) which becomes energetically unfavourable in small particles. For the system of $40 \mathrm{~nm}$ particles, phase stoichiometries are not stable indicating an enhanced solid solution behavior: lithium content increases to $\mathrm{Li}_{0.1} \mathrm{TiO}_{2}$ in $\mathrm{Li}$-poor and to $\mathrm{Li}_{0.7} \mathrm{TiO}_{2}$ in Li-rich phases. Further decrease of the particle size makes it possible to find a fully occupied phase $\mathrm{Li}_{1} \mathrm{TiO}_{2}$ [36]. It can coexist with the Li-rich one penetrating to the 3-4 $\mathrm{nm}$ depth below the particle surface (or transforming all the particles less than $7 \mathrm{~nm}$ in size). The poor Li ion conductivity can be due to the full occupation of the octahedral voids, whereas ion diffusion requires vacancies. Most likely, the short diffusion path in nanostructured materials diminishes this problem as well as elevation of temperature.

As established by quasi-elastic neutron scattering [37] Li ions can occupy two distinct positions within the octahedral interstices along the $c$ axis (but only one of them at a time). In the Li-anatase those positions are symmetrical, separated by $1.61 \AA$ and equally occupied while in the Li-titanate they are shifted, separated by only $0.7 \AA$ and nonequivalent $\left(n_{\mathrm{Li} 1}=0.32\right.$ and $n_{\mathrm{Li} 2}=0.19$ at $10 \mathrm{~K}$ - the fitting of the site occupancy assuming a Boltzmann distribution indicates that the energy difference between the positions is $3.8 \mathrm{meV}$; positions 1 and 2 have an antiparallel orientation in the neighbouring octahedra due to the phase symmetry). A combination of quasi-elastic neutron scattering and force field molecular dynamics simulations shows that $\mathrm{Li}$ is hopping on a picosecond time scale between the two sites in the octahedral interstices $[38,39]$.

Lithium was also found to occupy multiple positions inside the distorted oxygen octahedron of $\mathrm{Li}_{x} \mathrm{Mg}_{0.1} \mathrm{Ni}_{0.4} \mathrm{Mn}_{1.5} \mathrm{O}_{4}$ spinel [17]. Quite possible that this feature is common to a wide family of crystals with a similar structure but has been found only recently due to a higher precision of the experiment.

\section{Symmetry analysis of the lithium intercalated anatase: a possibility of internal piezoeffect}

As mentioned before, the standard crystallographic cell of body-centered anatase is chosen to consist of two elementary cells for convenience. Thus, the respective space group $D_{4 h}^{19}$ (or $I 4_{1}$ /amd origin choice 2 , No. 141) has a double set of symmetry operations as compared with its point symmetry group $D_{4 h}(4 / \mathrm{mmm})$. However, a single elementary cell obeys all transformation rules. It consists of two formula units, i.e. two octahedral voids (formed by oxygen anions) where the intercalated lithium ion can reside in one of two available positions (figure 1).

Occupation of each octahedron by the lithium ion could be easily described by the Hubbard projection operator $X_{i k}^{p p}$, where $i$ is the lattice site

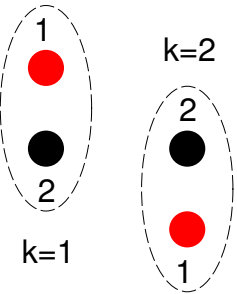

Figure 1. A schematic illustration of the positions available for the intercalated lithium ion in oxygen octahedron voids of the anatase elementary cell. Occupation of positions 1 and 2 is equal in the poor Li-anatase phase and different in the rich Li-titanate phase; their orientations are antiparallel in the neighbouring octahedra (sublattices $k=1,2$ ). 
index (i.e. the elementary cell index), $k=1,2$ is the sublattice index (the octahedron index), and the state $p=0$ corresponds to an empty octahedron while the states $p=1,2$ denote the lithium ion in positions 1 or 2 , respectively. The Hubbard operator formalism reflects the microscopic structure of the system and is very convenient for further calculations.

Alternatively, in the pseudospin formalism localization of lithium in a certain position can be described by the pseudospin operator $\hat{s}_{i k}=(-1)^{k-1}\left(X_{i k}^{11}-X_{i k}^{22}\right)$. However, one should also take into account the total occupation of the void $\hat{n}_{i k}=X_{i k}^{11}+X_{i k}^{22}$. Such an approach separates dipoledipole (pseudospin) and particle-particle interactions in the spirit of the Blume-Emery-Griffiths (BEG) model [40].

Finally, symmetrized linear combination of the averages

$$
n_{ \pm}=\frac{1}{2}\left(n_{1} \pm n_{2}\right), \quad \eta_{ \pm}=\frac{1}{2}\left(s_{1} \pm s_{2}\right), \quad\left(n_{k} \equiv\left\langle\hat{n}_{i k}\right\rangle, s_{k} \equiv\left\langle\hat{s}_{i k}\right\rangle\right)
$$

inherit symmetry properties of the system, thus being the order parameters of possible phase transitions (see appendix). Namely, $n_{+}$(it transforms according to the irreducible representation (IR) $A_{1 g}$ of the point group $D_{4 h}$ ) corresponds to the lithium concentration (the average occupation of octahedral voids), $n_{-}$(IR $B_{2 u}$ ) is the difference of the void occupations in the sublattices 1 and $2, \eta_{+}\left(\operatorname{IR} A_{2 u}\right)$ is the polarization along the $z$ axis, and $\eta_{-}$(IR $B_{1 g}$ which corresponds to the phase transition into the Li-titanate point symmetry subgroup $D_{2 h}$ ) simultaneously describes two phenomena: the antipolarization along the $z$ axis (unlike the true antiferroelectric ordering with doubling of the unit cell, a mutual compensation of sublattice polarizations occurs here just as in the Mitsui model) and the deformation $U_{x x}-U_{y y}$ in the $a b$ plane. Such a coexistence of the antipolar ordering and the deformation belonging to the same irreducible representation and, thus, described by a common order parameter is called internal piezoeffect.

\section{Phase equilibrium in the framework of the Landau expansion}

The symmetry analysis performed in the previous section can serve as a background for a qualitative description of thermodynamics of the considered system in the framework of Landau expansion

$$
F=F_{0}+\frac{1}{2} a \rho^{2}+\frac{1}{3} b \rho^{3}+\frac{1}{4} c \rho^{4}+\frac{1}{2} A \eta^{2}+\frac{1}{4} B \eta^{4}-\sigma \eta-\mu \rho,
$$

where $\rho$ describes the intercalant (lithium) concentration and hence corresponds to the $n_{+}$introduced above, $\mu$ is the chemical potential, the order parameter $\eta$ is proportional to the $\eta_{-}$, and the conjugated "field" $\sigma$ describes the applied stress; the expansion coefficients should satisfy the following conditions

$$
A=A_{0}+A_{1} \rho, \quad B>0, \quad c>0 .
$$

The equilibrium state of the system is achieved at the minimum of the free energy

$$
\begin{aligned}
& \frac{\partial F}{\partial \rho}=a \rho+b \rho^{2}+c \rho^{3}+\frac{1}{2} A_{1} \eta^{2}-\mu=0, \\
& \frac{\partial F}{\partial \eta}=A \eta+B \eta^{3}-\sigma=0
\end{aligned}
$$

Further calculations are limited to the case of zero "field": $\sigma=0$. Then, equation (4) can have either trivial solution $\eta=0$ or nonzero one:

$$
A+B \eta_{0}^{2}=0 \Rightarrow \eta_{0}= \pm \sqrt{-\left(A_{0}+A_{1} \rho\right) / B} .
$$

At $B>0, \eta_{0}$ takes on a real value under the condition $A_{0}+A_{1} \rho<0$ which means $A_{0}>0$ and $A_{1}<0$ giving

$$
\eta_{0}^{2}=\frac{\left|A_{1}\right| \rho-A_{0}}{B}, \quad \rho>\frac{A_{0}}{\left|A_{1}\right|} .
$$

Let us consider the cases $\eta=0$ and $\eta \neq 0$ separately. 
I. $\eta=0$

As follows from equation (3)

$$
\varphi(\rho)=\mu, \quad \varphi(\rho) \equiv a \rho+b \rho^{2}+c \rho^{3} .
$$

This equation could have three solutions in a certain region of chemical potential values (i.e. the possibility of a phase transition with the jump of $\rho$ ) if the extrema of the function $\varphi(\rho)$ exist, i.e. the equation

$$
\frac{\partial \varphi(\rho)}{\partial \rho}=a+2 b \rho+3 c \rho^{2}=0
$$

has nonzero solutions

$$
\rho_{1,2}=\frac{1}{3 c}\left[-b \pm \sqrt{b^{2}-3 a c}\right],
$$

which imposes a condition on the Landau expansion coefficients

$$
b^{2}-3 a c>0 .
$$

An equivalent condition could be obtained by setting the second derivative to zero

$$
\frac{\partial^{2} \varphi(\rho)}{\partial \rho^{2}}=2 b+6 c \rho=0,
$$

which gives the ordinate of the inflection point

$$
\rho^{*}=-b / 3 c,
$$

and demanding a negative value of the first derivative at this point $\partial \varphi(\rho) /\left.\partial \rho\right|_{\rho=\rho^{*}}<0$. Since the curve $\varphi(\rho)$ always crosses the inflection point (which is the symmetry centre of the curve), this point is also crossed by the line of the phase transition occurring at the following value of chemical potential

$$
\varphi\left(\rho^{*}\right)=\frac{b}{3 c}\left(\frac{2 b^{2}}{9 c}-a\right)=\mu^{*} .
$$

Considering that the parameter $\rho$ describes concentration, we have an additional condition $\rho \geqslant 0$ : both solutions of equation (8) are positive if $b>0$.

II. $\eta= \pm \eta_{0}$

After the identical calculations one can obtain an expression similar to equation (7) but slightly renormalized:

$$
\tilde{a} \rho+b \rho^{2}+c \rho^{3}=\tilde{\mu}, \quad \tilde{a}=a-\frac{\left|A_{1}\right|^{2}}{2 B}, \quad \tilde{\mu}=\mu-\frac{A_{0}\left|A_{1}\right|}{2 B} .
$$

The phase transition exists if

$$
b^{2}-3 \tilde{a} c>0
$$

and it occurs at the following value of chemical potential

$$
\tilde{\mu}^{*}=\frac{b}{3 c}\left(\frac{2 b^{2}}{9 c}-\tilde{a}\right) .
$$

As follows from the above considerations, the behaviour of the system at change of the chemical potential depends on the values of Landau expansion coefficients. So, further considerations are limited to the case which qualitatively describes the phase transition between the poor phase I $(\eta=0, \rho \rightarrow 0)$ and the rich phase II $(\eta \neq 0, \rho \rightarrow 0.5)$ in the lithiated anatase.

Comparing conditions (9) and (13) one can derive the condition

$$
3 a c-\frac{3}{2} \frac{\left|A_{1}\right|^{2}}{B} c<b^{2}<3 a c
$$


describing the case when only one solution for $\rho$ exists in the phase I while there are three possible solutions in the phase II at nonzero $\eta_{0}$. Combining the equations for equilibrium values of $\rho$ and the condition of the first order phase transition $F_{\mathrm{I}}=F_{\text {II }}$ between the phases I and II, we obtain a set of equations for values of $\rho_{\mathrm{I}}, \rho_{\mathrm{II}}$ and $\mu$ at the phase transition point

$$
\begin{aligned}
& a \rho_{\mathrm{I}}+b \rho_{\mathrm{I}}^{2}+c \rho_{\mathrm{I}}^{3}=\mu, \\
& \tilde{a} \rho_{\mathrm{II}}+b \rho_{\mathrm{II}}^{2}+c \rho_{\mathrm{II}}^{3}=\tilde{\mu}, \\
& \frac{1}{2} a \rho_{\mathrm{I}}^{2}+\frac{1}{3} b \rho_{\mathrm{I}}^{3}+\frac{1}{4} c \rho_{\mathrm{I}}^{4}-\mu \rho_{\mathrm{I}}=\frac{1}{2} a \rho_{\mathrm{II}}^{2}+\frac{1}{3} b \rho_{\mathrm{II}}^{3}+\frac{1}{4} c \rho_{\mathrm{II}}^{4}+\frac{1}{2} A\left(\rho_{\mathrm{II}}\right) \eta_{0}^{2}\left(\rho_{\mathrm{II}}\right)+\frac{1}{4} B \eta_{0}^{4}\left(\rho_{\mathrm{II}}\right)-\mu \rho_{\mathrm{II}} .
\end{aligned}
$$

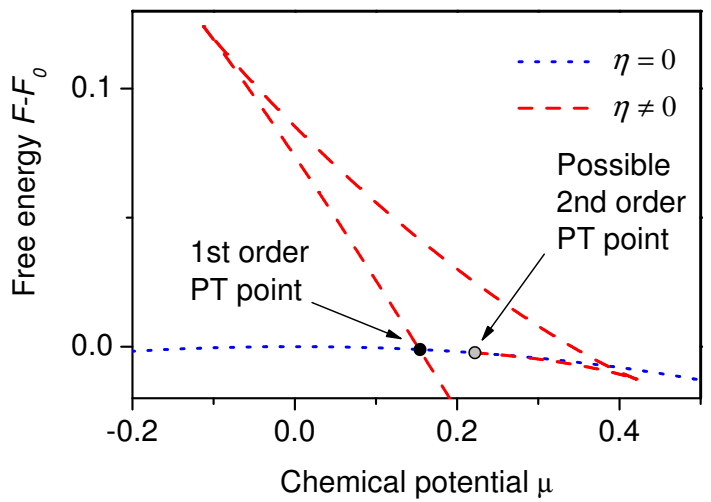

Figure 2. Dependence of free energy on chemical potential for branches with zero and nonzero order parameter $\eta$ : the first order phase transition takes place.

A qualitative illustration of such a phase transition, which corresponds to the case in the lithium intercalated anatase, is given in figure 2 for the following set of Landau coefficients (2): $a=10.8, b=-35.2, c=45.7, A_{0}=0.2$, $A_{1}=-9.1, B=9$. This set satisfies the condition (15) and, as follows from the free energy analysis, at the rise of chemical potential the first order phase transition from the branch I to the branch II (16) precedes the possible second order phase transition with a continuous growth of the nonzero value of $\rho$ at $\rho(\mu)=\rho_{c}$.

Due to the dependence on parameter $\rho$ of the coefficients of parameter $\eta$ both of them have simultaneous jumps at the point of the first order phase transition (figure 3). Parameter $\eta$ should be considered as a true order parameter of this transition because it is exactly equal to zero in the initial phase. It should be also noted that (due to proximity of the phase transition points) at increase of chemical potential the system could "pass through" the first order phase transition point (a metastable state) and the second order phase transition does occur followed by the first order phase transition in the extremum point of $\rho(\mu)$. All the above considerations are valid for the case $\mu=$ const. However, the lithiated anatase corresponds rather to the system with the fixed lithium concentration $(\rho=$ const). In this case the system separates into phases with concentrations $\rho_{\mathrm{I}}$ and $\rho_{\mathrm{II}}$ (figure 3 ) and respective weights $w_{\mathrm{I}}$ and $w_{\mathrm{II}}$, so $\rho_{\text {fixed }}=w_{\mathrm{I}} \rho_{\mathrm{I}}+w_{\mathrm{II}} \rho_{\mathrm{II}}$. The chemical potential of the system is constant and is equal to the chemical potential value at which the first order phase transition occurs in the $\mu=$ const regime.

Using the equality

$$
\frac{\partial^{2} F}{\partial \eta^{2}}=A+3 B \eta^{2}=\frac{\partial \sigma}{\partial \eta},
$$

one can calculate the susceptibility which describes the reaction of the order parameter $\eta$ (the deformation $U_{x x}-U_{y y}$ ) with respect to the "field" $\sigma$ (this susceptibility is related to the elastic modulus of the system)

$$
\chi \equiv \frac{\partial \eta}{\partial \sigma}=\left[A_{0}-\left|A_{1}\right| \rho+3 B \eta^{2}\right]^{-1} .
$$

Its explicit form depends on the phase

$$
\begin{array}{cl}
\eta=0: & \chi_{\mathrm{I}}=\left[A_{0}-\left|A_{1}\right| \rho_{\mathrm{I}}\right]^{-1}, \\
\eta=\eta_{0}: & \chi_{\mathrm{II}}=\frac{1}{2}\left[\left|A_{1}\right| \rho_{\mathrm{II}}-A_{0}\right]^{-1} .
\end{array}
$$

Due to the phase transition, the susceptibility has a jump whose value can be calculated from expressions (18) and (19) using solutions of the set (16). 

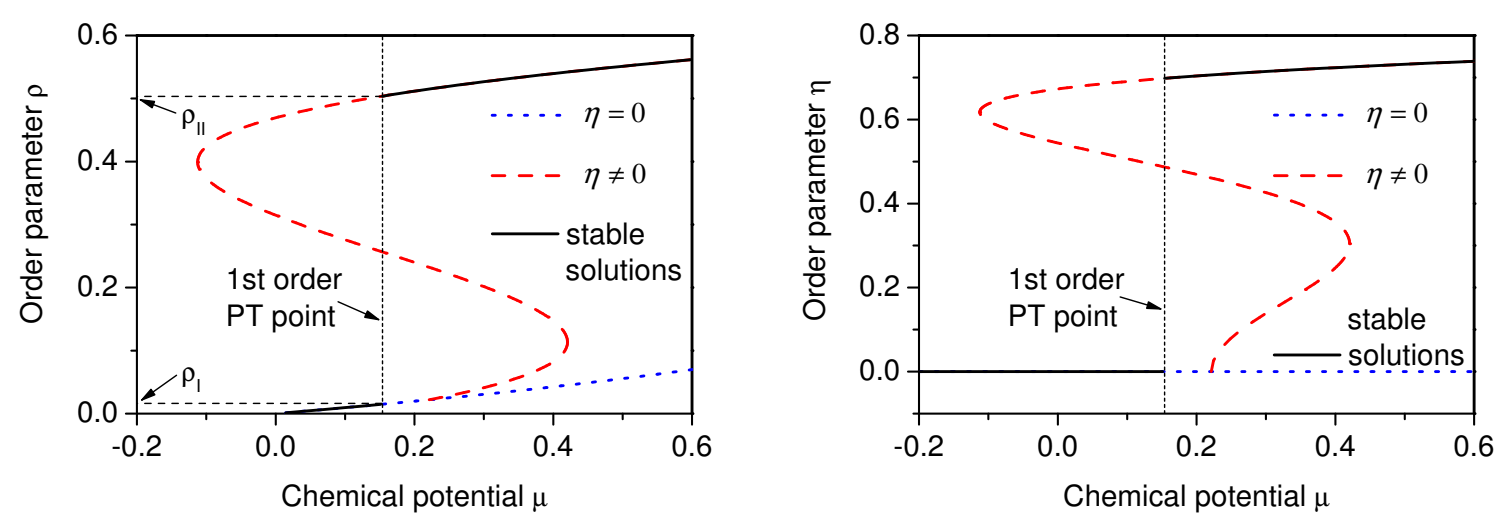

Figure 3. A jump of order parameters $\rho$ (left) and $\eta$ (right) at the first order phase transition. Thermodynamically stable solutions are marked with the solid curve. In the $\rho=$ const regime the phase separation into phases with $\rho_{\mathrm{I}}$ and $\rho_{\mathrm{II}}$ takes place.

Utilizing the equality $A_{0}-\left|A_{1}\right| \rho=-B \eta^{2}$, one can rewrite the susceptibility (17) at $\eta \neq 0$ in the form

$$
\chi=\left[2 B \eta^{2}\right]^{-1} .
$$

Thus, the susceptibility could diverge in both cases (18) and (20) approaching the point of the possible second order phase transition from the respective direction.

\section{Lattice model for the lithium intercalated anatase}

\subsection{Model Hamiltonian and thermodynamics in the mean field approximation}

As demonstrated in the previous section, the Landau expansion combined with the symmetry analysis gives a good qualitative picture of the phase separation in the lithiated anatase. However, a detailed description of temperature dependent thermodynamic properties of the system could be derived only in the framework of a microscopic approach. Let us construct a model Hamiltonian of the lattice gas type:

$$
\hat{H}=\hat{H}_{1}+\hat{H}_{\text {int }}+\hat{H}_{\text {def }},
$$

where besides the single particle term $\hat{H}_{1}$ the interparticle interaction $\hat{H}_{\text {int }}$ and the lattice deformation $\hat{H}_{\text {def }}$ are taken into account:

$$
\begin{aligned}
\hat{H}_{1} & =\sum_{i} \sum_{k} \sum_{p}\left(\varepsilon_{0}-\mu\right) X_{i k}^{p p}-h \sum_{i}\left(s_{i 1}+s_{i 2}\right)-\Delta \sum_{i}\left(s_{i 1}-s_{i 2}\right), \\
\hat{H}_{\mathrm{int}} & =-\frac{1}{2} \sum_{i \neq j} \sum_{k} \sum_{p q} W_{k k}^{p q}(i, j) X_{i k}^{p p} X_{j k}^{q q}, \\
\hat{H}_{\mathrm{def}} & =\frac{1}{2} N C U^{2}
\end{aligned}
$$

where

$$
\Delta=\alpha U, \quad U=U_{x x}-U_{y y} ; \quad k=1,2, \quad p=0,1,2
$$

$\mu$ is chemical potential of the intercalant particles (let us assign $\varepsilon_{0}$ as its origin), $h$ is the external electric field, $\Delta$ is the deformation induced internal field, $U$ is the effective deformation in the $X Y$ plane $\left(\sigma=\partial(\Omega / N) / \partial U\right.$ defines the stress), $W_{k k}^{p q}(i, j)$ are the interaction energies between the particles in the respective positions. Hence, we take into account both the semiphenomenological deformational shift of the lattice energy $\hat{H}_{\text {def }}$ and the effective internal staggered field $\Delta$ (similar to the one in the Mitsui model) which appears due to the intercalation induced lattice deformation making the lithium intercalation positions inequivalent in the pairs. Unlike the ordinary lattice gas 
approach, the proposed model considers two equilibrium positions for intercalated particles. Such multistate models (see, e.g. [41,42]) are rather rare since monopositional intercalated materials are the most common. Deformational effects have been also taken into account because an effective potential for the Li ions was affected by the intercalation-induced distortion of the host [43].

In the mean field approximation, Hamiltonian (21) becomes linear

$$
\hat{H}_{\mathrm{MFA}}=N E_{0}+\sum_{i} \sum_{k} \sum_{p} H_{k p} X_{i k}^{p p} .
$$

Taking into account the symmetry properties of the Fourier transforms of the interaction energies in the centre of the Brillouin zone

$$
\begin{array}{ll}
W_{11}^{11}=W_{11}^{22}=W_{22}^{11}=W_{22}^{22}, & W_{11}^{12}=W_{11}^{21}=W_{22}^{12}=W_{22}^{21}, \\
W_{12}^{12}=W_{21}^{21}=W_{12}^{21}=W_{21}^{12}, & W_{12}^{11}=W_{12}^{22}=W_{21}^{11}=W_{21}^{22},
\end{array}
$$

one can write down the expressions for average values and effective fields

$$
\begin{aligned}
E_{0}= & \frac{1}{2} W_{11}^{11}\left(\left\langle X_{1}^{11}\right\rangle^{2}+\left\langle X_{1}^{22}\right\rangle^{2}+\left\langle X_{2}^{11}\right\rangle^{2}+\left\langle X_{2}^{22}\right\rangle^{2}\right)+W_{11}^{12}\left(\left\langle X_{1}^{11}\right\rangle\left\langle X_{1}^{22}\right\rangle+\left\langle X_{2}^{11}\right\rangle\left\langle X_{2}^{22}\right\rangle\right) \\
& +W_{12}^{11}\left(\left\langle X_{1}^{11}\right\rangle\left\langle X_{2}^{11}\right\rangle+\left\langle X_{1}^{22}\right\rangle\left\langle X_{2}^{22}\right\rangle\right)+W_{12}^{12}\left(\left\langle X_{1}^{11}\right\rangle\left\langle X_{2}^{22}\right\rangle+\left\langle X_{1}^{22}\right\rangle\left\langle X_{2}^{11}\right\rangle\right), \\
H_{11}= & -\mu-h-\Delta-\left(W_{11}^{11}\left\langle X_{1}^{11}\right\rangle+W_{11}^{12}\left\langle X_{1}^{22}\right\rangle+W_{12}^{11}\left\langle X_{2}^{11}\right\rangle+W_{12}^{12}\left\langle X_{2}^{22}\right\rangle\right), \\
H_{12}= & -\mu+h+\Delta-\left(W_{11}^{11}\left\langle X_{1}^{22}\right\rangle+W_{11}^{12}\left\langle X_{1}^{11}\right\rangle+W_{12}^{11}\left\langle X_{2}^{22}\right\rangle+W_{12}^{12}\left\langle X_{2}^{11}\right\rangle\right), \\
H_{21}= & -\mu+h-\Delta-\left(W_{11}^{11}\left\langle X_{2}^{11}\right\rangle+W_{11}^{12}\left\langle X_{2}^{22}\right\rangle+W_{12}^{11}\left\langle X_{1}^{11}\right\rangle+W_{12}^{12}\left\langle X_{1}^{22}\right\rangle\right), \\
H_{22}= & -\mu-h+\Delta-\left(W_{11}^{11}\left\langle X_{2}^{22}\right\rangle+W_{11}^{12}\left\langle X_{2}^{11}\right\rangle+W_{12}^{11}\left\langle X_{1}^{22}\right\rangle+W_{12}^{12}\left\langle X_{1}^{11}\right\rangle\right) .
\end{aligned}
$$

Average occupations of the positions can be obtained as solutions of the selfconsistency equation set

$$
\left\langle X_{k}^{p p}\right\rangle=Z_{k}^{-1} \mathrm{e}^{-\beta H_{k p}},
$$

where partition functions of sublattices are as follows:

$$
Z_{k}=1+\mathrm{e}^{-\beta H_{k 1}}+\mathrm{e}^{-\beta H_{k 2}}
$$

and thermodynamically stable solutions are chosen according to the criterion of the minimum of grand canonical potential

$$
\Omega / N=E_{0}+\frac{1}{2} C U^{2}-\Theta \ln \left(Z_{1} Z_{2}\right) .
$$

For the $(n, s)$-representation (introduced in section 2) selfconsistency equations look like

$$
\begin{aligned}
n_{k} & =Z_{k}^{-1}\left(\mathrm{e}^{-\beta H_{k 1}}+\mathrm{e}^{-\beta H_{k 2}}\right), \\
s_{k} & =(-1)^{k-1} Z_{k}^{-1}\left(\mathrm{e}^{-\beta H_{k 1}}-\mathrm{e}^{-\beta H_{k 2}}\right),
\end{aligned}
$$

where the term $E_{0}$ and the effective fields are as follows

$$
\begin{aligned}
E_{0} & =\frac{1}{4}\left[W_{11}^{+}\left(n_{1}^{2}+n_{2}^{2}\right)+W_{11}^{-}\left(s_{1}^{2}+s_{2}^{2}\right)+2 W_{12}^{+} n_{1} n_{2}-2 W_{12}^{-} s_{1} s_{2}\right], \\
H_{11} & =-\mu-h-\Delta-\frac{1}{2}\left(W_{11}^{+} n_{1}+W_{11}^{-} s_{1}+W_{12}^{+} n_{2}-W_{12}^{-} s_{2}\right), \\
H_{12} & =-\mu+h+\Delta-\frac{1}{2}\left(W_{11}^{+} n_{1}-W_{11}^{-} s_{1}+W_{12}^{+} n_{2}+W_{12}^{-} s_{2}\right), \\
H_{21} & =-\mu+h-\Delta-\frac{1}{2}\left(W_{12}^{+} n_{1}+W_{12}^{-} s_{1}+W_{11}^{+} n_{2}-W_{11}^{-} s_{2}\right), \\
H_{22} & =-\mu-h+\Delta-\frac{1}{2}\left(W_{12}^{+} n_{1}-W_{12}^{-} s_{1}+W_{11}^{+} n_{2}+W_{11}^{-} s_{2}\right),
\end{aligned}
$$

and new combinations of interaction energies are introduced

$$
W_{11}^{ \pm}=W_{11}^{11} \pm W_{11}^{12}, \quad W_{12}^{ \pm}=W_{12}^{11} \pm W_{12}^{12} .
$$


Finally, the symmetrized combinations of averages (1) (which can be the order parameters of the system) are found from the following set

$$
\begin{aligned}
& n_{ \pm}=\frac{1}{2}\left[Z_{1}^{-1}\left(\mathrm{e}^{-\beta H_{11}}+\mathrm{e}^{-\beta H_{12}}\right) \pm\left(\mathrm{e}^{-\beta H_{21}}+\mathrm{e}^{-\beta H_{22}}\right)\right] \\
& \eta_{ \pm}=\frac{1}{2}\left[Z_{1}^{-1}\left(\mathrm{e}^{-\beta H_{11}}-\mathrm{e}^{-\beta H_{12}}\right) \mp\left(\mathrm{e}^{-\beta H_{21}}-\mathrm{e}^{-\beta H_{22}}\right)\right]
\end{aligned}
$$

with the respective definitions for $E_{0}$ and the effective fields

$$
\begin{aligned}
E_{0} & =\frac{1}{2}\left[W_{++} n_{+}^{2}+W_{+-} n_{-}^{2}+W_{--} \eta_{+}^{2}+W_{-+} \eta_{-}^{2}\right], \\
H_{11} & =-\mu-h-\Delta-\frac{1}{2}\left(W_{++} n_{+}+W_{+-} n_{-}+W_{--} \eta_{+}+W_{-+} \eta_{-}\right), \\
H_{12} & =-\mu+h+\Delta-\frac{1}{2}\left(W_{++} n_{+}+W_{+-} n_{-}-W_{--} \eta_{+}-W_{-+} \eta_{-}\right), \\
H_{21} & =-\mu+h-\Delta-\frac{1}{2}\left(W_{++} n_{+}-W_{+-} n_{-}-W_{--} \eta_{+}+W_{-+} \eta_{-}\right), \\
H_{22} & =-\mu-h+\Delta-\frac{1}{2}\left(W_{++} n_{+}-W_{+-} n_{-}+W_{--} \eta_{+}-W_{-+} \eta_{-}\right),
\end{aligned}
$$

as well as symmetrized interaction energies

$$
W_{+ \pm}=W_{11}^{+} \pm W_{12}^{+}, \quad W_{- \pm}=W_{11}^{-} \pm W_{12}^{-}
$$

Considering the definition $\sigma=\partial(\Omega / N) / \partial U$ with account of the equality $\Delta=\alpha U$ and the expression for grand canonical potential (27), the equation for the deformation is obtained

$$
U=\frac{2 \alpha}{C}\left(\tilde{\sigma}+\eta_{-}\right)
$$

where $\tilde{\sigma}=\sigma / 2 \alpha$ is a scaled dimensionless stress. As it follows

$$
\frac{1}{2} C U^{2}=k_{\Delta}\left(\tilde{\sigma}+\eta_{-}\right)^{2}, \quad \Delta=k_{\Delta}\left(\tilde{\sigma}+\eta_{-}\right)
$$

where $k_{\Delta}=2 \alpha^{2} / C$. Thus, the deformation (34) can occur spontaneously (giving rise to the antisymmetrical internal field $\Delta$ ) due to the appearance of the order parameter $\eta_{-}$even at the absence of the stress.

The deformation $U$ is a proper variable for the grand canonical potential $\Omega(27)$ but in our case it is convenient to deal with the stress $\sigma$ (conjugated to $U$ ). Performing the Legendre transformation

$$
\mathrm{d} \Omega=\cdots+\sigma \mathrm{d} U=\cdots+\mathrm{d}(\sigma U)-U \mathrm{~d} \sigma
$$

one can build the desired form of grand canonical potential $\widetilde{\Omega}$

$$
\widetilde{\Omega}=\Omega-\sigma U
$$

The respective deformational term of the thermodynamic potential looks like

$$
\frac{1}{2} C U^{2}-\sigma U=k_{\Delta}\left(\eta_{-}^{2}-\tilde{\sigma}^{2}\right)
$$

\subsection{Phase diagram of the ground state}

At zero temperature, the homogenious system, which is described by Hamiltonian (21), could reside in one of the nine possible states $\left|p_{1} p_{2}\right\rangle$ (let us also use a more descriptive notation where "up" and "down" arrows indicate occupied positions, e.g. $|10\rangle \equiv|\uparrow 0\rangle)$ with the following values of 
thermodynamic potential (36):

$$
\begin{aligned}
& |00\rangle \equiv|0\rangle: \quad \widetilde{\Omega}_{0}=-k_{\Delta} \tilde{\sigma}^{2}, \\
& |\uparrow 0\rangle \equiv|1\rangle: \quad \widetilde{\Omega}_{1}=-\mu-h-W_{1}-k_{\Delta}\left(\tilde{\sigma}+\frac{1}{2}\right)^{2} \text {, } \\
& |\downarrow 0\rangle \equiv|2\rangle: \quad \quad \widetilde{\Omega}_{2}=-\mu+h-W_{1}-k_{\Delta}\left(\tilde{\sigma}-\frac{1}{2}\right)^{2} \text {, } \\
& |0 \downarrow\rangle \equiv|3\rangle: \quad \widetilde{\Omega}_{3}=-\mu+h-W_{1}-k_{\Delta}\left(\tilde{\sigma}+\frac{1}{2}\right)^{2} \text {, } \\
& |0 \uparrow\rangle \equiv|4\rangle: \quad \widetilde{\Omega}_{4}=-\mu-h-W_{1}-k_{\Delta}\left(\tilde{\sigma}-\frac{1}{2}\right)^{2} \text {, } \\
& |\uparrow \uparrow\rangle \equiv|5\rangle: \quad \widetilde{\Omega}_{5}=-2 \mu-2 h-2 W_{2}-k_{\Delta} \tilde{\sigma}^{2}, \\
& |\downarrow \downarrow\rangle \equiv|6\rangle: \quad \widetilde{\Omega}_{6}=-2 \mu+2 h-2 W_{2}-k_{\Delta} \tilde{\sigma}^{2}, \\
& |\uparrow \downarrow\rangle \equiv|7\rangle: \quad \quad \widetilde{\Omega}_{7}=-2 \mu-2 W_{3}-k_{\Delta}(\tilde{\sigma}+1)^{2} \text {, } \\
& |\downarrow \uparrow\rangle \equiv|8\rangle: \quad \quad \widetilde{\Omega}_{8}=-2 \mu-2 W_{3}-k_{\Delta}(\tilde{\sigma}-1)^{2} \text {; }
\end{aligned}
$$

where

$$
\begin{aligned}
& W_{1}=\frac{1}{8}\left(W_{++}+W_{+-}+W_{--}+W_{-+}\right)=\frac{1}{2} W_{11}^{11}, \\
& W_{2}=\frac{1}{4}\left(W_{++}+W_{--}\right)=\frac{1}{2}\left(W_{11}^{11}+W_{12}^{12}\right), \\
& W_{3}=\frac{1}{4}\left(W_{++}+W_{-+}\right)=\frac{1}{2}\left(W_{11}^{11}+W_{12}^{11}\right) .
\end{aligned}
$$

As is obvious from expressions (37), at $\tilde{\sigma}>0$ (in particular at $\tilde{\sigma} \rightarrow+\varepsilon$ ) the levels 1,3 , and 7 always lie below the levels 4, 2 and 8, respectively. So, the latter will not be further considered.

Setting equal thermodynamic potentials of different phases, one can obtain a set of equations for the respective phase transitions:

$$
\begin{array}{ll}
|0\rangle \leftrightarrow|1\rangle: & \mu=-h-W_{1}-k_{\Delta}\left(\tilde{\sigma}+\frac{1}{4}\right), \\
|0\rangle \leftrightarrow|3\rangle: & \mu=h-W_{1}-k_{\Delta}\left(\tilde{\sigma}+\frac{1}{4}\right), \\
|0\rangle \leftrightarrow|5\rangle: & \mu=-h-W_{2}, \\
|0\rangle \leftrightarrow|6\rangle: & \mu=h-W_{2}, \\
|0\rangle \leftrightarrow|7\rangle: & \mu=-W_{3}-k_{\Delta}\left(\tilde{\sigma}+\frac{1}{2}\right), \\
|1\rangle \leftrightarrow|3\rangle: & h=0, \\
|5\rangle \leftrightarrow|6\rangle: & h=0, \\
|5\rangle \leftrightarrow|7\rangle: & \mu=\left(W_{3}-W_{2}\right)+k_{\Delta}\left(\tilde{\sigma}+\frac{1}{2}\right), \\
|6\rangle \leftrightarrow|7\rangle: & \mu=-\left(W_{3}-W_{2}\right)-k_{\Delta}\left(\tilde{\sigma}+\frac{1}{2}\right), \\
|1\rangle \leftrightarrow|5\rangle: & \mu=-h+\left(W_{1}-2 W_{2}\right)+k_{\Delta}\left(\tilde{\sigma}+\frac{1}{4}\right), \\
|3\rangle \leftrightarrow|6\rangle: & \mu=h+\left(W_{1}-2 W_{2}\right)+k_{\Delta}\left(\tilde{\sigma}+\frac{1}{4}\right), \\
|1\rangle \leftrightarrow|7\rangle: & \mu=h+\left(W_{1}-2 W_{3}\right) k_{\Delta}\left(\tilde{\sigma}+\frac{3}{4}\right), \\
|3\rangle \leftrightarrow|7\rangle: & \mu=3 h+\left(W_{1}-2 W_{2}\right)+k_{\Delta}\left(\tilde{\sigma}+\frac{1}{4}\right), \\
|1\rangle \leftrightarrow|6\rangle: & \mu=-3 h+\left(W_{1}-2 W_{2}\right)+k_{\Delta}\left(\tilde{\sigma}+\frac{1}{4}\right) . \\
|3\rangle \leftrightarrow|5\rangle: & \mu
\end{array}
$$

Various possible phase diagrams of the ground state in the $\mu-h$ plane (which are calculated from equations (39)) are depicted in figures 4-7 using the following designations:

$$
\begin{aligned}
\mu_{0} & =-W_{3}-k_{\Delta}\left(\tilde{\sigma}+\frac{1}{2}\right), \\
\mu_{1} & =-W_{1}-k_{\Delta}\left(\tilde{\sigma}+\frac{1}{4}\right), \\
\mu_{2} & =W_{1}-2 W_{3}-k_{\Delta}\left(\tilde{\sigma}+\frac{3}{4}\right), \\
\mu_{3} & =W_{1}-2 W_{2}+k_{\Delta}\left(\tilde{\sigma}+\frac{1}{4}\right), \\
h_{1} & =W_{3}-W_{2}+k_{\Delta}\left(\tilde{\sigma}+\frac{1}{2}\right) .
\end{aligned}
$$




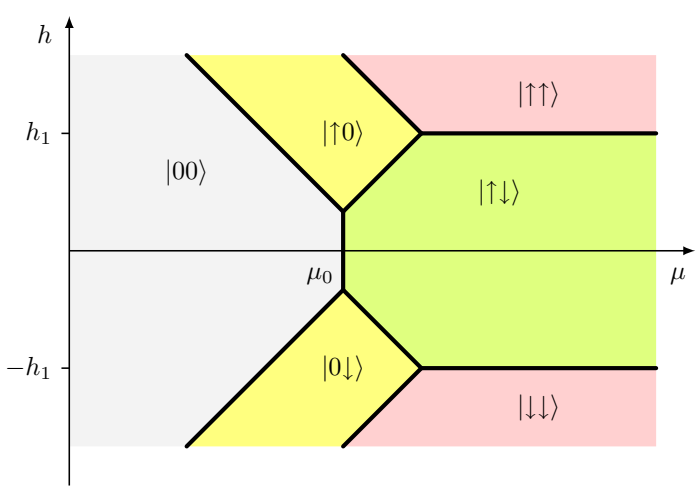

Figure 4. The most general form of the ground state phase diagram: existence of intermediate half-filled phases and the possibility of a direct transition from the empty state to the full one.

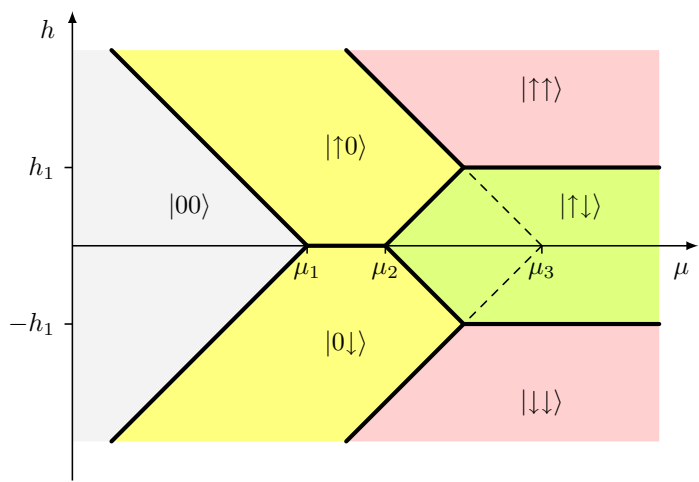

Figure 5. The ground state phase diagram with the phase transition between the empty and half-filled states which corresponds to the Li-poor - Li-rich phase equilibrium in the lithiated anatase.

It is evident that the form of the phase diagram depends on the values of model parameters, so we shall analyse some important cases below.

In the most general case (figure 4), the phase diagram consists of empty, half-filled, and full states which differ in occupation and polarization of sublattices. Two-sublattice nature of the model demonstrates itself in the intermediate half-filled states and the central nonpolar full state thus being noticeably different from the respective ground state diagram of the BEG model. It should be stressed that due to the exclusion of the states 2,4 , and 8 the phase $|\uparrow 0\rangle \equiv|1\rangle$ is symmetrical to the phase $|0 \downarrow\rangle \equiv|3\rangle$. In the considered case, both transitions between the empty and half-filled phases as well as between the empty and full phases are possible. The latter transition takes place, e.g. at zero field $h$ which is contrary to the situation in the lithiated anatase where only half-filled phases are accessible.

Coexistence of the Li-poor and Li-rich phases in the lithiated anatase is fairly described by a phase diagram in figure 5. For any value of the external electric field $h$ (in particular, at zero field) the system can pass from the empty state to the half-filled state only. In the regime of fixed concentration such a phase transition manifests itself as a phase separation.
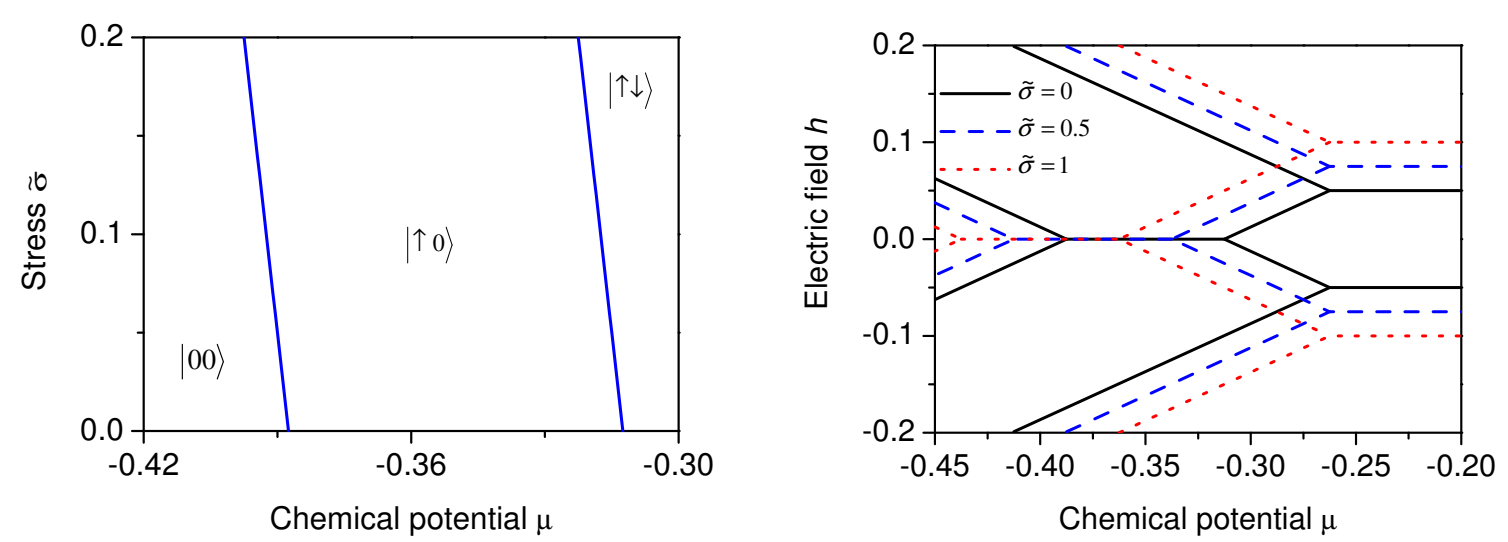

Figure 6. The effect of stress imposition: the ground state phase diagram in the $\mu-\tilde{\sigma}$ coordinates at $h=0$ (left) and a family of ground state phase diagrams for different values of the stress (right). Other model parameters have the following values: $W_{++}=1, W_{+-}=1.5, W_{--}=0.2$, $W_{-+}=0.3, k_{\Delta}=0.05$. 
Such a phase diagram exists if conditions $\mu_{1}<\mu_{2}<\mu_{3}$ are satisfied. Taking into account definitions (40) and (38) and neglecting deformational effects one can rewrite the above inequality in a more clear form

$$
W_{12}^{12}<W_{12}^{11}<0
$$

Thus, this type of a phase diagram exists if the interactions between the orientational states in different states are repulsive with the interaction between the unlike states (with different $p$, i.e. $|\uparrow 0\rangle$ and $|0 \uparrow\rangle$ or $|\downarrow 0\rangle$ and $|0 \downarrow\rangle)$ being stronger than the interaction between the like states (with identical $p$, i.e. $|\uparrow 0\rangle$ and $|0 \downarrow\rangle$ or $|\downarrow 0\rangle$ and $|0 \uparrow\rangle)$. These conclusions are in full qualitative agreement with the predictions of the molecular dynamics modelling [37].

In view of deformational effects, the inequalities $\mu_{1}<\mu_{2}<\mu_{3}$ transform to

$$
-k_{\Delta}\left(\tilde{\sigma}+\frac{1}{4}\right)<-W_{12}^{11}-k_{\Delta}\left(\tilde{\sigma}+\frac{3}{4}\right)<-W_{12}^{12}+k_{\Delta}\left(\tilde{\sigma}+\frac{1}{4}\right) .
$$

Thus, application of the stress $\tilde{\sigma}$ favours the phase diagram in figure 5 because $\mu_{1}$ and $\mu_{2}$ are shifted to the left $\sim \tilde{\sigma}$ and $\mu_{3}$ is moved towards the right $\sim \tilde{\sigma}$. So the domains of the phases $|\uparrow 0\rangle$ and $|0 \downarrow\rangle$ expand. However, at $h=0$ the difference $\mu_{2}-\mu_{1}=-W_{12}^{11}-\frac{1}{2} k_{\Delta}$ does not depend on the stress

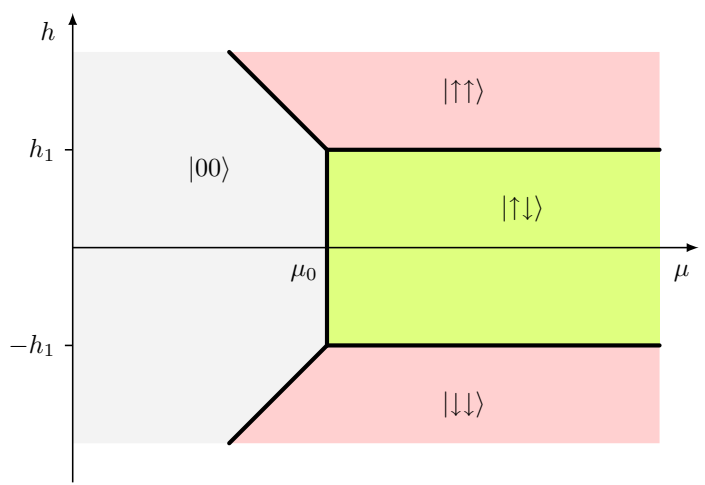

Figure 7. A reduced form of the ground state phase diagram: only the empty and full phases coexist.

(as one can see on the phase diagram $\mu-\tilde{\sigma}$ in figure 6) and half-filled phases exist if $W_{12}^{11}<-\frac{1}{2} k_{\Delta}$. A strong enough stress $\tilde{\sigma}$ can "open" these phases even if conditions (41) are not satisfied. It is easily seen from inequalities (42) that the appearance of spontaneous deformation also favours the expansion of the half-filled domains (even at $\tilde{\sigma}=0$ ). The above rationales are confirmated by the family of phase diagrams in figure 6 calculated for different stress values $\tilde{\sigma}$ (hereinafter all model parameters are given in the dimensionless units normalized by $W_{++}=1$; in the considered case $W_{11}^{11}=0.75, W_{11}^{12}=0.5, W_{12}^{11}=-0.1$, $W_{12}^{12}=-0.15$ in such a manner satisfying conditions (41)).

If conditions (42) fail, the half-filled phases are suppressed (figure 7 ). In addition, if $h_{1} \leqslant 0$, then the central full nonpolar phase vanishes and the ground state phase diagram reduces to the respective one for the BEG model.

\subsection{Temperature behaviour of phase diagrams and phase separation}

Since the considered model inherits features of the Mitsui and BEG models, one can expect a quite complex thermodynamical behaviour and the above analysis of the ground state has proved these anticipations. As it is seen from the temperature axis complemented analogue (figure 8) of the diagram in figure 5, the lines of phase transitions form the surfaces and some new phases appear. A comprehensive analysis of the obtained diagram is too complicated and goes beyond the scope of this research, so we consider the case with intermediate halffilled phases corresponding to the intercalated anatase.

With this in mind let us analyse the phase diagram at the absence of the external electric

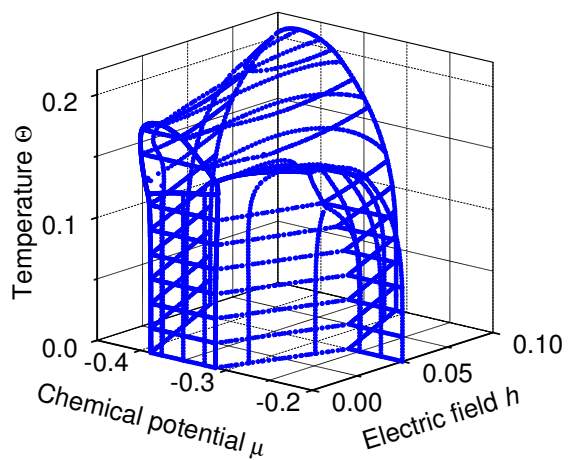

Figure 8. The three-dimensional phase diagram in the $\mu-h-\Theta$ coordinates. Model parameters have the following values: $W_{++}=1, W_{+-}=1.5$, $W_{--}=0.2, W_{-+}=0.3, k_{\Delta}=0.05, \tilde{\sigma}=0$. 
field $h$ (figure 9). At low temperature, in full agreement with the ground state diagram, there are three phases: "empty", "half-filled", and "full" (due to the temperature "blurring" at higher temperatures these names loose their exact meaning). At high temperatures in the whole range of chemical potential there is only "empty" phase where all order parameters are zero except the concentration $n_{+}$which changes monotonously.

The "half-filled" phase provides the most complex behaviour of the order parameters. As is seen from the "cross-section" at the chemical potential value $\mu=-0.38$ (figure 10 ), the rise of temperature leads to the suppression of the dipole-dipole ordering (order parameters $\eta_{+}$ and $\left.\eta_{-}\right)$and it completely vanishes at the line of the second order phase transition which is located inside the "half-filled" phase. The phase itself is limited from the top side by the line of the phase transition with zeroing of $n_{-}$. The upper part of the phase is separated by the tricritical points (TCPs) marking the change of the phase transition order from the second to the first one. The "full" phase is also limited from the top side by the line of the second order transitions where $\eta_{-} \rightarrow 0$.

Behaviour of order parameters at the change of chemical potential (figure 10) clearly distinguishes the phases separated by the lines of the first order phase transitions (e.g. a characteristic feature of the "half-filled" phase is $n_{-} \neq 0$ ). In a certain temperature range inside the "half-filled"

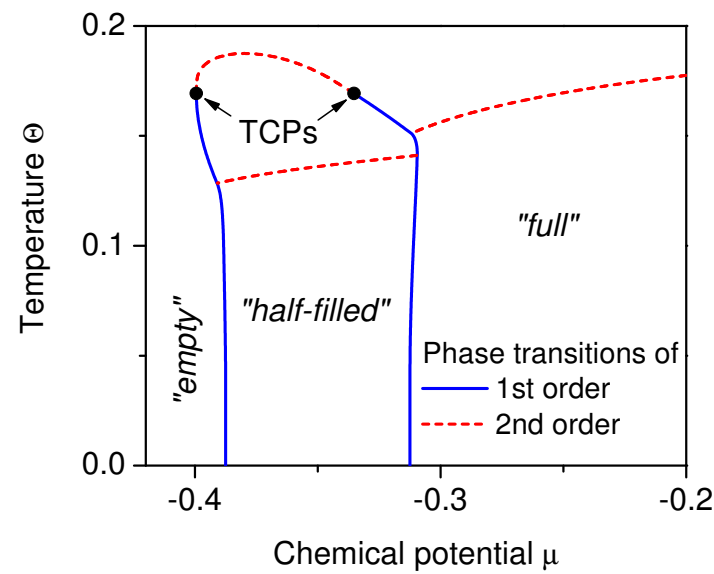

Figure 9. The phase diagram $\mu-\Theta$ at the absence of the electric field. Other model parameters are as follows: $W_{++}=1, W_{+-}=1.5$, $W_{--}=0.2, W_{-+}=0.3, k_{\Delta}=0.05, \tilde{\sigma}=$ 0 . Phase names ("empty", "half-filled", and "full") correspond to the ground state. There are two tricritical points (TCPs) on the phase transition line limiting the "half-filled" phase from the top. phase, the above mentioned phase transition between the polar and non-polar states takes place.

In a wide temperature range, the appearance of the phase diagram "chemical potential $\mu-$ stress $\tilde{\sigma} "$ (figure 11) closely resemble the ground state one (figure 6). However, the further rise of temperature leads to a fast suppression of the "half-filled" phase.
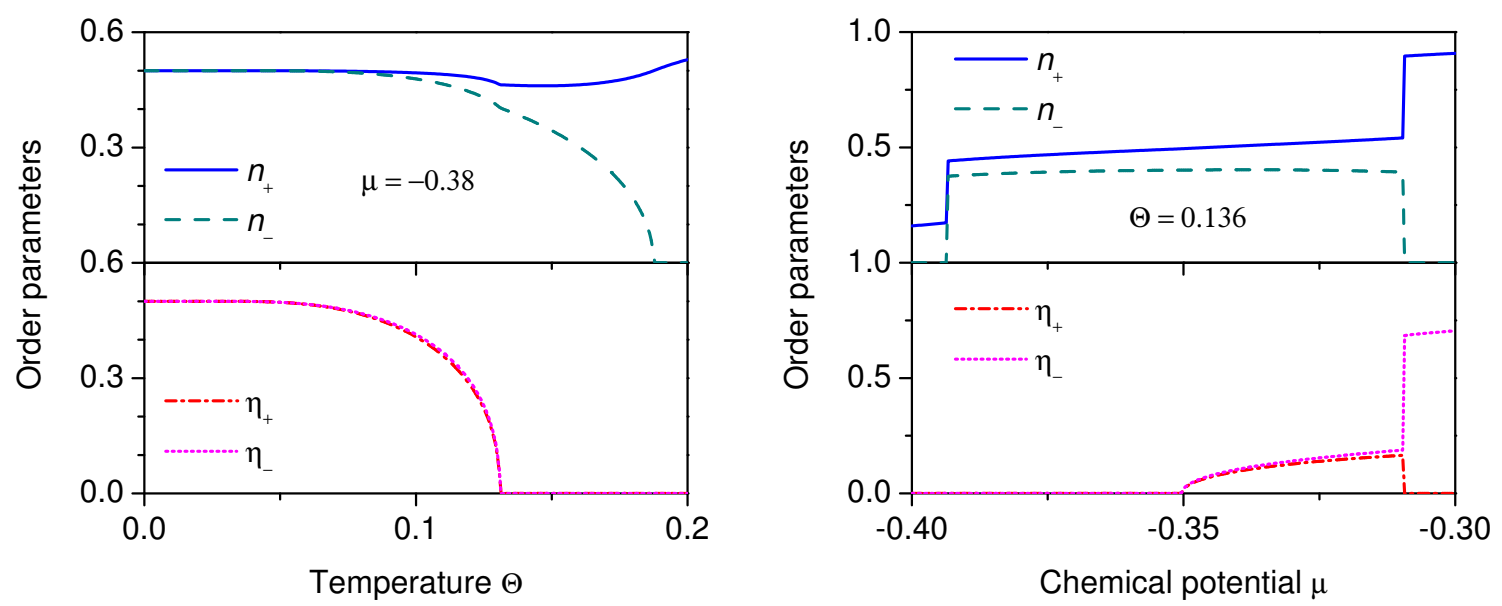

Figure 10. Phase diagram "cross-sections": temperature dependences of order parameters in the "half-filled" phase (left: $\mu=-0.38$; in the picture scale curves $\eta_{+}$and $\eta_{-}$overlap) and dependences of order parameters on chemical potential exhibiting phase transitions of the second and the first orders (right: $\Theta=0.136$ ). Other model parameters have the following values: $W_{++}=1, W_{+-}=1.5, W_{--}=0.2, W_{-+}=0.3, k_{\Delta}=0.05, \tilde{\sigma}=0$. 


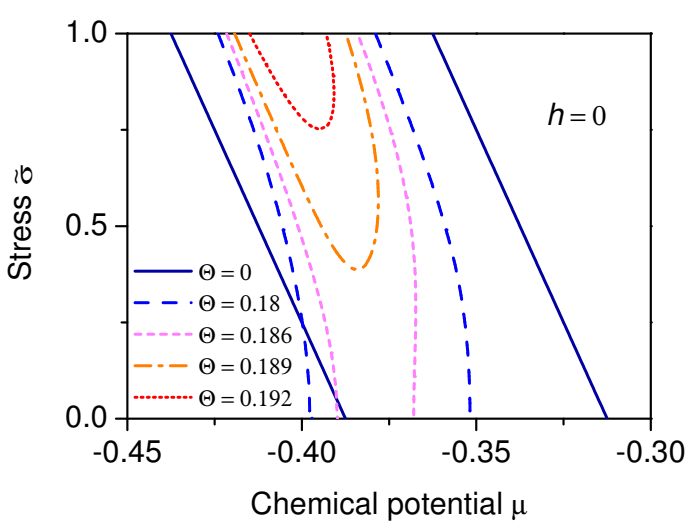

Figure 11. A family of phase diagrams "chemical potential $\mu$-stress $\tilde{\sigma}$ " for various temperature values at $h=0$. Other model parameters have the following values: $W_{++}=1, W_{+-}=$ $1.5, W_{--}=0.2, W_{-+}=0.3, k_{\Delta}=0.05$.

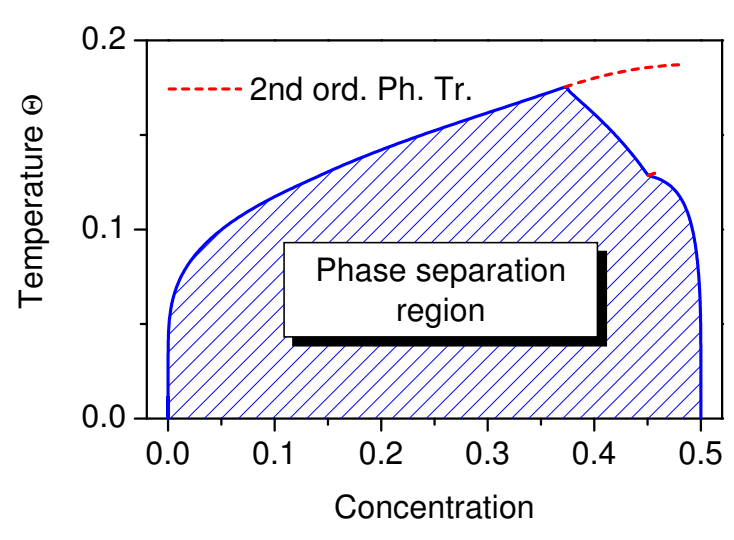

Figure 12. The diagram of the phase separation into "poor" $\left(n_{+}=0\right)$ and "rich" $\left(n_{+}=0.5\right)$ phases in the regime $n_{+}=$const $\left(W_{++}=1, W_{+-}=1.5, W_{--}=0.2, W_{-+}=\right.$ $0.3, k_{\Delta}=0.05, \tilde{\sigma}=0$ ).

Since in the regime of a fixed chemical potential $(\mu=$ const) the "empty" and "half-filled" phases on the phase diagram $\mu-\Theta$ (figure 9) are separated mainly by the line of the first order phase transitions, the system separates into "poor" and "rich" phases (figure 12) in the regime of the fixed concentration $\left(n_{+}=\right.$const). As one can see, in a wide region of low temperatures a separation into concentrations $n_{+}=0$ and $n_{+}=0.5$ occurs which well reproduces the coexistence of Li-poor and Li-rich phases in the intercalated anatase. The phase separation region narrows at heating and finally closes up at the point corresponding to the tricritical point in figure 9. Another short line of phase transitions (starting at the kink of the right boundary) relates to the suppression of polar states in a "half-filled" phase.

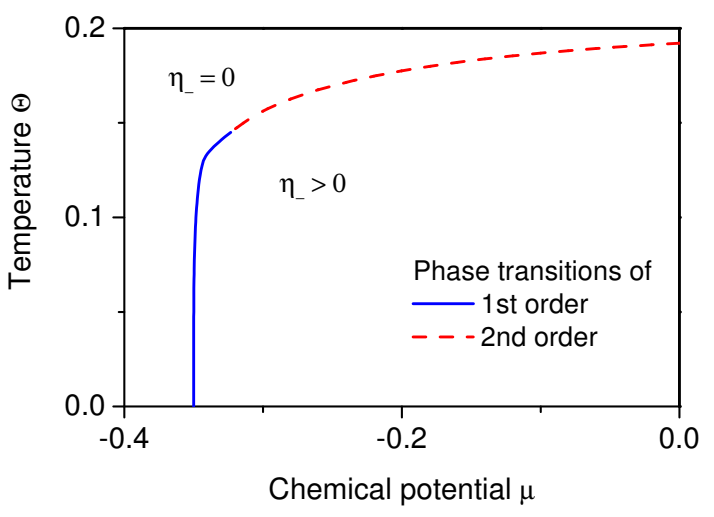

Figure 13. The phase diagram $\mu-\Theta$ at $h=0$ for the case of the first order phase transition between "empty" and "full" phases $\left(n_{-}=0\right.$ and $\eta_{+}=0$ in both of them; $W_{++}=1, W_{+-}=0$, $\left.W_{--}=0, W_{-+}=0.3, k_{\Delta}=0.05, \tilde{\sigma}=0\right)$.
A question arises why in the microscopic model we should deal with four order parameters while the Landau expansion is quite successful with only two? First of all, the semiphenomenological description is qualitative only and it just demonstrates the possibility of the first order phase transition with simultaneous jumps of concentration and the order parameter $\eta$ as the minimal set necessary to describe the phase separation in the litiated anatase. Such a picture corresponds to a direct phase transition between the "empty" and "full" phases (see the phase diagram at zero external field in figure 9) when other order parameters $\left(n_{-}\right.$and $\left.\eta_{+}\right)$are always equal to zero. The dependences of the "active" order parameters $n_{+}$and $\eta_{-}$on chemical potential (figure 14) closely resemble the respective curves for $\rho$ and $\eta$ obtained by the Landau expansion (figure 3 ). But if one should take into account the "half-

filled" phase (what is inevitable for description of the lithiated anatase), the order parameters $\left(n_{-}\right.$ and $\left.\eta_{+}\right)$became nonzero and the full set of four parameters should be considered as it has been done above. 

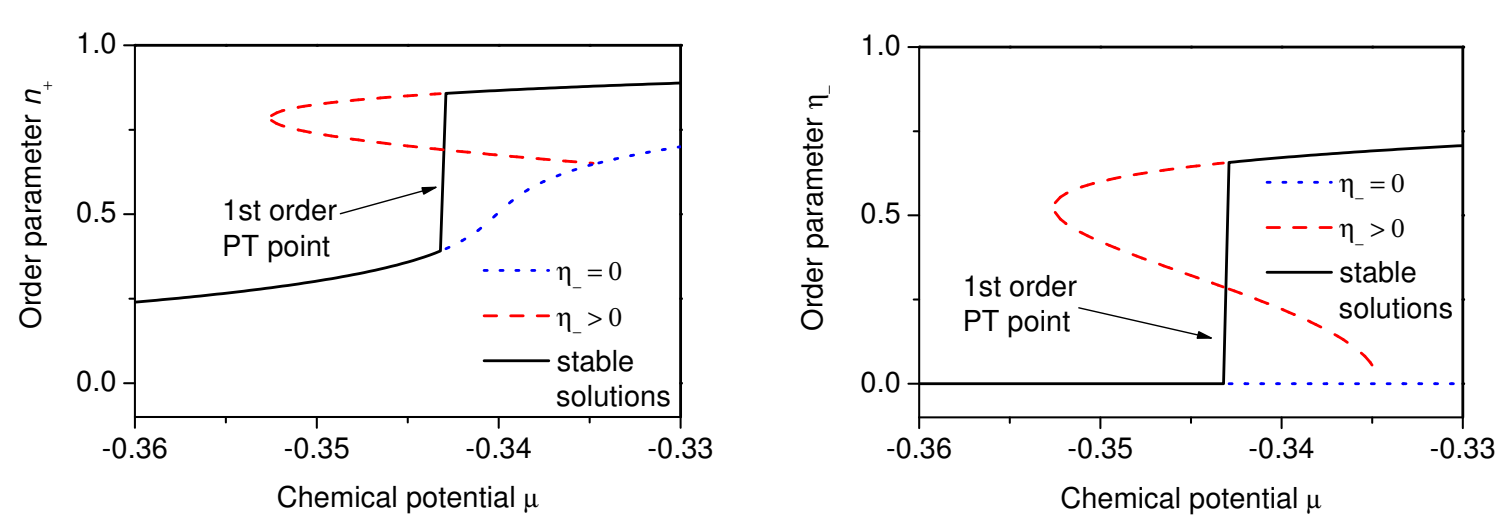

Figure 14. A jump of order parameters $n_{+}$(left) and $\eta_{-}$(right) at the first order phase transition illustrating the previous phase diagram. Thermodynamically stable solutions are marked with the solid curve. Other model parameters have the following values: $W_{++}=1, W_{+-}=0, W_{--}=$ $0, W_{-+}=0.3, k_{\Delta}=0.05, \Theta=0.13, h=0, \tilde{\sigma}=0$.

\section{Conclusions}

To sum up, the present study was inspired by two features of the lithium intercalated anatase: coexistence of poor and rich phases and two possible localizations of $\mathrm{Li}$ ion in the oxygen octahedron along the $c$ axis. The second one implies the possibility of (anti)polar ordering what is beyond the scope of a simple lattice gas model well describing a phase separation. So a model of Blume-EmeryGriffits type has been used which takes into account both particle-particle and dipole-dipole terms.

Another less obvious peculiarity of the considered compound is the intercalation induced deformation of lattice: Li-rich phase has a lower symmetry (the axes $a$ and $b$ become inequivalent) resulting in preferential occupation of one position of the mentioned pairs which has an alternating orientation in the neighbour octahedra (i.e. in different sublattices). Performed symmetry analysis explains this phenomena by the possibility of internal piezoeffect: the deformation in the $a b$ plane as well as the appearance of an effective internal staggered field (causing the ordering of antiferroelectric type like as in the Mitsui model) both belong to the same irreducible representation of the initial high-symmetry anatase phase and, hence, are described by the common order parameter. Thus, increase of the intercalant content could result in a phase transition with simultaneous jumps of the average occupation and antipolarization (the latter accompanied by the jump of dielectric susceptibility) as it has been proved by the Landau expansion.

The microscopic approach, combining the abovementioned features of both the BEG and Mitsui models, gives semiquantitative description of phase coexistence in the lithiated anatase. Analysis of the ground state phase diagram confirms a possibility of the phase transition between "empty" and "half-filled" phases which corresponds to the phase separation into Li-poor and Li-rich phases in the crystal. As the model predicts, such a separation remains near constant in a wide temperature range. The microscopic approach could easily reproduce the Landau expansion results as the particular case of the "empty" - "full" transition described by the two order parameters. But the presence of the "half-filled" phase makes it necessary to deal with the full set of the order parameters allowed by the crystal symmetry.

However, some issues are still open. The model predicts that external stress should shift the phase transition between empty and half-filled phases to the lower values of chemical potential. An experimental evidence of this conjecture is still missing. The real average occupation in the Li-rich phase is $0.55-0.6$ instead of the value $n_{+}=0.5$ in the half-filled model phase. This deviation could be explained by a multidomain nature of the Li-rich phase containing "impurities" of the full-occupied $\mathrm{LiTiO}_{2}$ phase while the model phases are monodomain by definition. The same explanation applies to the issue of absence of the total polarization in the half-filled phases: at zero external field these phases with opposite polarizations could coexist in different domains providing a full mutual compensation. A similar mechanism of the mutual compensation of po- 
larization in Li- "chains" with an opposite Li orientation is supported by the molecular dynamics simulations [37].

\section{Acknowledgements}

Oleh Velychko is deeply indebted to the project "Improvement of functional possibilities of the Western coordinating grid-centre of Ukrainian Academic Grid (the City of Lviv)" for financial support and a possibility to perform numerical calculations on its cluster.

\section{Appendix: Table of the point group $D_{4 h}$ symmetry transformations for the symmetrized averages}

Table 1. Transformations of the symmetrized linear combinations of the averages which correspond to the irreducible representations (IR) of the point symmetry group $D_{4 h}$. The operations, which also belong to the lower symmetry subgroup $D_{2 h}$, are marked by asterisk; they keep the symmetrized combination $\eta_{-}$(IR $B_{1 g}$ ) invariant.

\begin{tabular}{|c||c|r|r|r|r|r|r|r|}
\hline IR & ${ }^{*} E$ & ${ }^{*} C_{2}^{(z)}$ & $C_{4}$ & $C_{4}^{3}$ & ${ }^{*} C_{2}^{(y)}$ & ${ }^{*} C_{2}^{(x)}$ & $C_{2}^{(x y)}$ & $C_{2}^{(x \bar{y})}$ \\
\hline \hline$A_{1 g}$ & $n_{+}$ & $n_{+}$ & $n_{+}$ & $n_{+}$ & $n_{+}$ & $n_{+}$ & $n_{+}$ & $n_{+}$ \\
$B_{2 u}$ & $n_{-}$ & $n_{-}$ & $-n_{-}$ & $-n_{-}$ & $-n_{-}$ & $-n_{-}$ & $n_{-}$ & $n_{-}$ \\
$A_{2 u}$ & $\eta_{+}$ & $\eta_{+}$ & $\eta_{+}$ & $\eta_{+}$ & $-\eta_{+}$ & $-\eta_{+}$ & $-\eta_{+}$ & $-\eta_{+}$ \\
$B_{1 g}$ & $\eta_{-}$ & $\eta_{-}$ & $-\eta_{-}$ & $-\eta_{-}$ & $\eta_{-}$ & $\eta_{-}$ & $-\eta_{-}$ & $-\eta_{-}$ \\
\hline
\end{tabular}

\begin{tabular}{|c||r|r|r|r|r|r|r|r|}
\hline IR & ${ }^{*} I$ & ${ }^{*} m_{(x y)}$ & $S_{4}^{3}$ & $S_{4}$ & ${ }^{*} m_{(x z)}$ & ${ }^{*} m_{(y z)}$ & $m_{(x \bar{y})}$ & $m_{(x y)}$ \\
\hline \hline$A_{1 g}$ & $n_{+}$ & $n_{+}$ & $n_{+}$ & $n_{+}$ & $n_{+}$ & $n_{+}$ & $n_{+}$ & $n_{+}$ \\
$B_{2 u}$ & $-n_{-}$ & $-n_{-}$ & $n_{-}$ & $n_{-}$ & $n_{-}$ & $n_{-}$ & $-n_{-}$ & $-n_{-}$ \\
$A_{2 u}$ & $-\eta_{+}$ & $-\eta_{+}$ & $-\eta_{+}$ & $-\eta_{+}$ & $\eta_{+}$ & $\eta_{+}$ & $\eta_{+}$ & $\eta_{+}$ \\
$B_{1 g}$ & $\eta_{-}$ & $\eta_{-}$ & $-\eta_{-}$ & $-\eta_{-}$ & $\eta_{-}$ & $\eta_{-}$ & $-\eta_{-}$ & $-\eta_{-}$ \\
\hline
\end{tabular}

\section{References}

1. Cangiani G., Baldereschi A., Posternak M., Krakauer H., Phys. Rev. B, 2004, 69, 121101.

2. Fujishima A., Honda K., Nature, 1972, 238, 37.

3. Wang R., Hashimoto K., Fujishima A., Chikuni M., Kojima E., Kitamura A., Shimohigoshi M., Watanabe T., Nature, 1997, 388, 431.

4. Earle M.D., Phys. Rev., 1942, 61, 56.

5. Savage N., Chwieroth B., Ginwalla A., Patton B.R., Akbar S.A., Dutta P.K., Sensors and Actuators B: Chemical, 2001, 79, 17.

6. Wu J.M., Chen C.J., J. Am. Ceram. Soc., 1990, 73, 420.

7. Brown W.D., Grannenmann W.W., Solid State Electron., 1978, 21, 837.

8. Badwey W.A., Mantaz R.S., Afify H.H., Elgiar E.M., J. Mater. Sci. Mater. Electron., 1991, $2,112$.

9. Siefering K.L., Griffin G.L., J. Electrochem. Soc., 1990, 137, 1206.

10. Tang H., Berger H., Schmid P., Lévy F., Solid State Commun., 1994, 92, 267.

11. Grätzel M., Comments on Inorganic Chemistry, 1991, 12, 93.

12. Kay A., Grätzel M., Solar Energy Materials and Solar Cells, 1996, 44, 99.

13. Bechinger C., Ferrere S., Zaban A., Sprague J., Gregg B.A., Nature, 1996, 383, 608.

14. Huang S.Y., Kavan L., Exnar I., Grätzel M., Journal of The Electrochemical Society, 1995, 142, L142.

15. Wagemaker M., Ven A.V.D., Morgan D., Ceder G., Mulder F., Kearley G., Chemical Physics, 2005, 317, 130.

16. Rho Y.H., Kanamura K., Journal of Power Sources, 2006, 158, 1436.

17. Wagemaker M., Ooms F.G.B., Kelder E.M., Schoonman J., Mulder F.M., Journal of the American Chemical Society, 2004, 126, 13526.

18. Cava R.J., Murphy D.W., Zahurak S., Santoro A., Roth R.S., Journal of Solid State Chemistry, 1984, $\mathbf{5 3}, 64$.

19. Stashans A., Lunell S., Bergström R., Hagfeldt A., Lindquist S.E., Phys. Rev. B, 1996, 53, 159. 
20. Mackrodt W.C., Journal of Solid State Chemistry, 1999, 142, 428.

21. Aydinol M.K., Kohan A.F., Ceder G., Cho K., Joannopoulos J., Phys. Rev. B, 1997, 56, 1354.

22. Koudriachova M.V., Harrison N.M., de Leeuw S.W., Phys. Rev. Lett., 2001, 86, 1275.

23. Koudriachova M.V., Harrison N.M., de Leeuw S.W., Phys. Rev. B, 2002, 65, 235423.

24. Koudriachova M.V., Harrison N.M., de Leeuw S.W., Solid State Ionics, 2002, 152-153, 189.

25. Koudriachova M.V., Harrison N.M., de Leeuw S.W., Computational Materials Science, 2002, 24, 235.

26. Koudriachova M.V., Harrison N.M., de Leeuw S.W., Solid State Ionics, 2003, 157, 35.

27. Koudriachova M.V., de Leeuw S.W., Harrison N.M., Chemical Physics Letters, 2003, 371, 150.

28. Koudriachova M.V., Harrison N.M., de Leeuw S.W., Solid State Ionics, 2004, 175, 829.

29. Koudriachova M., Chemical Physics Letters, 2008, 458, 108.

30. Caravaca M.A., Miño J.C., Pérez V.J., Casali R.A., Ponce C.A., Journal of Physics: Condensed Matter, 2009, 21, 015501.

31. Mysakovych T., Stasyuk I., Journal of Physical Studies, 2007, 11, 1327.

32. Wagemaker M., van de Krol R., Kentgens A.P.M., van Well A.A., Mulder F.M., Journal of the American Chemical Society, 2001, 123, 11454.

33. Wagemaker M., Kentgens A., Mulder F., Nature, 2002, 418, 397.

34. Wagemaker M., van de Krol R., van Well A.A., Physica B: Condensed Matter, 2003, 336, 124.

35. Wagemaker M., Borghols W.J.H., van Eck E.R.H., Kentgens A.P.M., Kearley G.J., Mulder F.M., Chemistry - A European Journal, 2007, 13, 2023.

36. Wagemaker M., Borghols W.J.H., Mulder F.M., Journal of the American Chemical Society, 2007, 129, 4323.

37. Wagemaker M., Kearley G.J., van Well A.A., Mutka H., Mulder F.M., Journal of the American Chemical Society, 2003, 125, 840.

38. Wagemaker M., van Well A., Kearley G., Mulder F., Solid State Ionics, 2004, 175, 191.

39. Gubbens P., Wagemaker M., Sakarya S., Blaauw M., Yaouanc A., de Réotier P.D., Cottrell S., Solid State Ionics, 2006, 177, 145.

40. Blume M., Emery V., Griffiths R., Phys. Rev. A, 1971, 4, 1071.

41. McKinnon W., Haering R. - In: Modern aspects of electrochemistry, vol. 15, Acad. Press, New-York, London, 1983, 235-304.

42. Grygorchak I., Voitovych S., Stasyuk I., Velychko O., Menchyshyn O., Condens. Matter Phys., 2007, 10, 51 .

43. Vakarin E., Badiali J., Levi M., Aurbach D., Phys. Rev. B, 2000, 63, 014304.

\title{
Фазове розшарування в інтеркальованому літієм анатазі: теорія
}

\author{
О.В.Величко, І.В.Стасюк \\ Інститут фізики конденсованих систем НАН України, 79011 Львів, вул. І. Свєнціцького, 1
}

Отримано 25 травня 2009 р., в остаточному вигляді - 26 травня 2009 р.

\begin{abstract}
Для інтеркальованого літієм анатазу, що використовується у літій-іонних батереях, характерні співіснування багатої та бідної на літій фаз та наявність двох можливих позицій для літію у кисневих октаедрах. Запропоновано теоретичний опис цієї сполуки, що враховує згадані особливості. Як показав проведений симетрійний аналіз, викликана інтеркаляцією деформація гратки може супроводжуватися впорядкуванням антисегнетоелектричного типу (внутрішній п'єзоефект). Це дало можливість отримати якісну ілюстрацію фазового розшарування у літійованому анатазі за допомогою розкладу Ландау з відповідним вибором коефіцієнтів. Для опису даної сполуки запропоновано також мікроскопічну модель, яка поєднує риси моделей Міцуї та Блюма-Емері-Гріффітса та використовує результати симетрійного аналізу. Різноманітні фазові діаграми моделі (як для основного стану, так i температурно-залежні) досліджувались з метою встановити набір модельних параметрів, що відповідає літійованому анатазу. В широкому температурному діапазоні виявлено фазове розшарування на порожню та напівзаповнену фази, що цілком відповідає співіснуванню фаз у інтеркальованому кристалі. В рамках моделі двопозиційна підсистема літію може мати впорядкування сегнето- чи антисегнетоелектричного типу, яке, однак, наразі експериментально не спостерігалося.
\end{abstract}

Ключові слова: анатаз, інтеркаляція, літій, фазове розшарування, розклад Ландау, граткова модель

PACS: $71.20 . T x, 64.60 . C n, 64.60 . D e$ 